\title{
Influence of Sodium Alginate Concentration on Microcapsules Properties Foreseeing the Protection and Controlled Release of Bioactive Substances
}

\author{
Kamal Essifi $\mathbb{D}^{\mathbb{D}},{ }^{1}$ Mohamed Brahmi ${ }^{\mathbb{D}},{ }^{1}$ Doha Berraaouan $\left(\mathbb{D},{ }^{1}\right.$ Abderrahim Ed-Daoui ${ }^{\mathbb{D}},{ }^{2}$ \\ Ali El Bachiri $\mathbb{1}^{1},{ }^{1}$ Marie-Laure Fauconnier $\mathbb{1}^{1},{ }^{3}$ and Abdesselam Tahani $\mathbb{1}^{1}$ \\ ${ }^{1}$ Physical Chemistry of Natural Substances and Process Team, \\ Laboratory of Applied Chemistry and Environment (LCAE-CPSUNAP), Department of Chemistry, Faculty of Sciences, \\ University Mohamed I, Oujda, Morocco \\ ${ }^{2}$ Laboratory of Theoretical Physics, Particles Modeling and Energetic, URAC 07, Faculty of Science, University Mohammed I, \\ Oujda, Morocco \\ ${ }^{3}$ Laboratory of Chemistry of Natural Molecules of Gembloux Agro-Bio Tech, University of Liège, Liège, Belgium
}

Correspondence should be addressed to Kamal Essifi; kamal.essifi.lpapc@gmail.com and Abdesselam Tahani; abdesstaha@ gmail.com

Received 6 July 2021; Accepted 16 August 2021; Published 21 August 2021

Academic Editor: Jorge F. Fernandez-Sanchez

Copyright (C) 2021 Kamal Essifi et al. This is an open access article distributed under the Creative Commons Attribution License, which permits unrestricted use, distribution, and reproduction in any medium, provided the original work is properly cited.

To understand the abilities of Ca-alginate microcapsules and their specific applications in different fields, it is necessary to determine the physicochemical and structural properties of those formulated microcapsules. In this work, we aimed to study the effect of alginate concentration in the improvement of the encapsulation efficiency (EE) and on the release of phenolic and flavonoid substances. The relationship between the structure of the encapsulated bioactive substance and Ca-alginate network and their effect on the EE and release kinetics have been investigated. The incorporation, structure, morphology, and phase properties of all elaborated materials were characterized by UV-spectroscopy, Fourier transform infrared (ATR-FTIR), scanning electron microscope (SEM), and X-ray diffraction (DRX). The results indicate that increasing the polymer concentration increases the EE and decreases the loading capacity (LC), whereas the effect of alginate polymer concentration on the release was not observed. The release study of bioactive substances showed that the release kinetics is relatively dependent on the structure and the physicochemical characteristics of the bioactive substance, which became clear when the encapsulated compounds were released from the core of calcium alginate microcapsules. Thus, it could be concluded that the pores size of the Ca-alginate network is smaller than the volume of the crocin molecule $\left(2794.926 \AA^{3}\right)$ and higher than the volume of the gallic acid molecule $\left(527.659 \AA^{3}\right)$. For the same microcapsules system, the release mechanism is affected by the structure and physicochemical properties of the encapsulated molecules.

\section{Introduction}

Microencapsulation technology aims to protect bioactive substances, during processing, improving stability from external harsh conditions in final products and during processing, masking undesirable odors and tastes of ingredients, and the delivery of the encapsulated matter at controlled rates, at a certain time, and in a certain place $[1,2]$. The choice of shell material is an important step in order to encapsulate a bioactive substance. Generally, the mechanical and physicochemical characteristics of a microcapsule are directly linked to the shell structure which is easily affected by the structure and the thickness of the wall material, the chemical composition, and the size of the microcapsule $[1,3]$.

Alginate acid extracted from brown algae is a natural biopolysaccharide which has many advantages compared to commonly used biopolymers for hydrogel beads 
formulation [4-6]. The mannuronic acid (M-block), guluronic acid (G-block), and mixed sequences of M-Gblocks are the main chains that constitute the alginate polymer. The sequence and ratio $(\mathrm{M} / \mathrm{G})$ of these uronic acids vary with the origin of alginate and control their properties [7]. A broad range of application can be found in food and pharmaceutical industries due to the biocompatibility, biodegradability, and nontoxicity of this biomaterial and also to their gelling properties when it crosslinked with divalent ions such as calcium and ferrous cation [3, 8-10]. Sodium alginate polymer, obtained from brown algae, has a number of features that makes it particularly appropriate to form hydrogel microcapsules, such as its low cost, high availability, biodegradability, biocompatibility with other agents, and easy synthesis of microparticles $[3,8,11,12]$.

The application area of alginate microcapsules is mainly depending on the properties presented by those microcapsules, which make it necessary to determine their physicochemical, mechanical, and structural properties. Alginate microcapsules are largely applied in the pharmaceutical industries, as an example, due to their ability to release the bioactive substance through the diffusion mechanism. On the other hand, another important mechanical property is the release rate, which is mainly related to the porosity of the microcapsules. The release of the encapsulated substance in different applications is mostly due to the diffusion mechanism, as a principal process in controlled release systems. The diffusion mechanism can be shortened into the transport of bioactive substance from the internal core to the external microcapsule shell [13-17].

During the release, the microcapsule matrix might remain unchanged, but in some cases, the change can occur due to the swelling behavior, shrinking, or fragmentation phenomena. Other factors can control the release of bioactive substance beside the structural properties of the microcapsules, such as the structure of those bioactive compounds and the physicochemical properties of the encapsulating material like their solubility degree in the microcapsules internal core, rheological properties presented by the substance inside the polymer capsule, the pore size of biopolymer microcapsules, the potential interaction between the microcapsule network and the encapsulated substance microcapsules properties (such as shape, size, and structure), and the concentration gradient of bioactive substance between the microcapsules wall and its surrounding environment [13-18]. The relative importance of those different parameters depends on the composition and internal structure of the elaborated microparticles.

In our previously reported work [3] on the mechanical properties of calcium alginate microcapsules, the increase of sodium alginate concentration led to the improvement of the mechanical strength of microcapsules. In the present study, we mainly focused on the properties of microcapsules elaborated with different sodium alginate concentrations foreseeing the encapsulation and controlled release of bioactive phenolic and flavonoid substances such as gallic acid and crocin as bioactive substances which have different structures and physicochemical characteristics. In addition, to better understand the relationship between the structure of encapsulated bioactive substances and the structure of microcapsules network, the effect of sodium alginate concentration and the structure of bioactive substances on the encapsulation efficiency, loading capacity, and the release was also examined.

\section{Materials and Methods}

2.1. Materials. In this study, a medium viscosity (5-40 cps of $1 \%$ aqueous solution) sodium alginate, with a molecular weight of $22500 \mathrm{~g} / \mathrm{mol}$, purchased from Sigma-Aldrich Co. (St. Louis, USA) was used.

Crocin, gallic acid monohydrate $(\geq 99 \%)$, calcium chloride dihydrate, and trisodium citrate were obtained from Sigma-Aldrich Co. (St. Louis, USA) and used without any further purification.

The conductivity of the distilled water used for all sample preparation was less than $2 \mu \mathrm{S}$.

\subsection{Preparing Calcium Alginate Microcapsules. Calcium} alginate microcapsules containing bioactive substances were prepared using the ionotropic gelation method under optimal formulation as described previously in our works [8]. Briefly, 3-6\% (w/v) of the sodium alginate and 1.5\% (w/v) of bioactive substance were dissolved in $100 \mathrm{ml}$ of distilled water. Hydration of samples was assured using magnetic stirring. After full hydration using a magnetic stirring, the air bubbles formed during the mixing were removed from the aqueous medium by allowing the solution to stand for two hours. The mixed solution was left to stand for about two hours to remove air bubbles from the aqueous medium. The gelling-bath solution was prepared by dissolving $\mathrm{CaCl}_{2}$ in distilled water $4 \%(\mathrm{w} / \mathrm{v})$. A pump was applied to control the delivery of the mixed solution of sodium alginate and bioactive substance through a tube which was connected with a polypropylene micropipette tip, and the mixture solution falls into the $\mathrm{CaCl}_{2}$ solution continuously, as shown in Figure 1.

2.3. Bioactive Substances Characteristics. The characteristics and the geometry optimization of bioactive substances were carried out using MOPAC package, module version 2016, and the calculation was performed with the RM1 semiempirical method.

2.4. Bioactive Substance Content Measurement and Loading Efficiency. The amount of BS was determined by the direct measurement of the absorbance using the UV-visible spectrophotometer at $265 \mathrm{~nm}$ for GA and $440 \mathrm{~nm}$ for crocin.

(i) For the gallic acid, the concentration was obtained through a calibration curve using GA as the standard reference, under identical experimental conditions

(ii) For the crocin agent, the concentration was obtained through a calibration curve using crocin as the standard reference, under the similar experimental conditions 


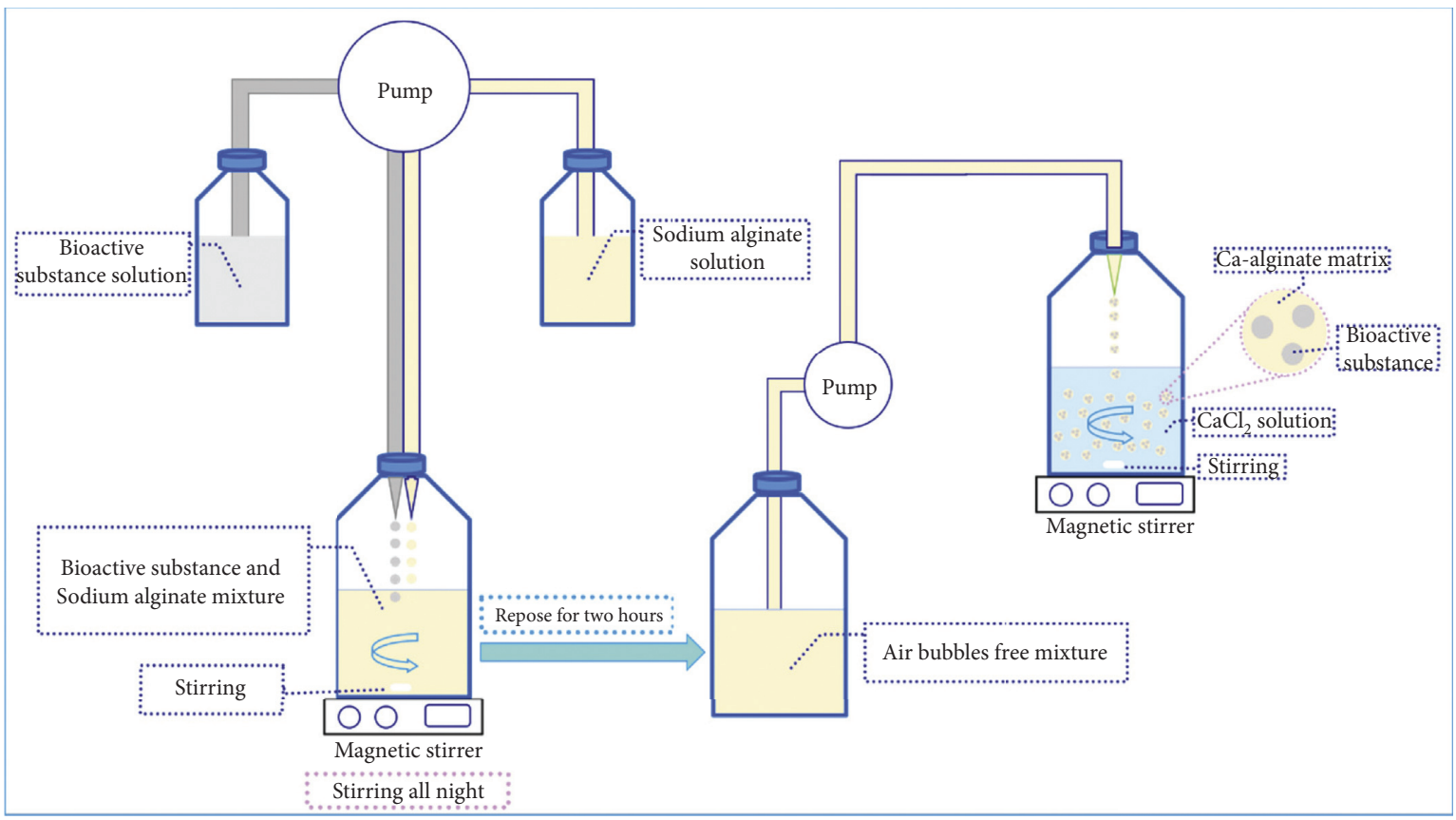

Figure 1: Simple schematic illustration for the preparation of bioactive substances loaded in Ca-alginate microcapsules.

Loading capacity was calculated using the following formula:

$$
\text { loading capacity }=\frac{\text { weight of bioactive agent in the calcium alginate microbeads }}{\text { weight of dried calcium alginate microbeads }} \times 100 \text {. }
$$

The encapsulation efficiency was calculated using the following formula:

$$
\text { encapsulation efficiency }=\frac{\text { amount of bioactive agent in the beads }}{\text { original amount of bioactive agent }} \times 100 \text {. }
$$

\subsection{Attenuated Total Reflection Fourier Transform Infrared} (ATR-FTIR) Analysis. The interactions between calcium alginate microcapsule and the encapsulated bioactive substance have been studied using the ATR-FTIR technique. The spectrums were carried out on a Jasco4700-ATR spectrophotometer (Shimadzu, Japan), in the wavelength region between 400 and $4000 \mathrm{~cm}^{-1}$. The obtained spectra for each sample were affected by averaging 32 scans at a resolution of $4 \mathrm{~cm}^{-1}$.

2.6. Morphological Characterization. The external structure of the obtained calcium alginate microcapsules was evaluated using a scanning electron microscope (FEI ESEM "Quanta 200," USA). The scanned micrographs were collected digitally by ETD software.
2.7. X-Ray Analysis. To investigate the physical form (amorphous or crystalline) of the blank and gallic acid loaded microcapsules, X-ray analysis were performed. The X-ray diffraction patterns were acquired on a Shimadzu XRD-6000 (Shimadzu, Japan), using the $\mathrm{CuK} \alpha$ radiation. The patterns were collected in $2 \theta$ range of $2^{\circ}-80^{\circ}$ with a step size of $0.02^{\circ}$.

2.8. GA Release Study. The release of bioactive substances from the elaborated microparticles in an aqueous medium was achieved using the UV-visible spectrophotometer. The analysis was realized based on our previously described method [8]. Briefly, a known amount of microcapsules was suspended in $100 \mathrm{ml}$ of distilled water. The samples underwent a continuous agitation on an multiposition 
magnetic stirrer (Variomag Poly 15, Germany) operating at $250 \mathrm{rpm}$. An aliquot of the supernatant was taken at predetermined time intervals for absorbance measurement at 265 and $440 \mathrm{~nm}$, respectively, for gallic acid and crocin, and the released bioactive substances amount was obtained by using concentration versus absorbance calibration curves.

Finally, $30 \mathrm{ml}$ of a sodium citrate solution $(2 \%$, w/v) was added, and final absorbance was recorded and corrected by considering the dilution.

2.9. Analysis of Kinetics Release Data. Zero-order, first-order, and Korsmeyer-Peppas mathematical models were carried out to evaluate the kinetic release behavior of the encapsulated compounds from alginate microcapsule in the interest to predict and correlate their release $[19,20]$.

Zero-order model is

$$
Q=k_{0} t+Q_{0} .
$$

$Q$ in the model equation represents the amount of the active compound released as function of time $t$ ( $\mathrm{min})$, $Q_{0}$ is the initial value $Q$, whereas $k_{0}$ is a rate constant. First-order model is

$$
Q=Q_{0} e^{k_{1} t}
$$

$Q$ in the model equation represents the amount of the active compound released as function of time $t$ ( $\mathrm{min})$, $Q_{0}$ is the initial value of $Q$, whereas $k_{1}$ is a rate constant.

Korsmeyer-Peppas model is

$$
Q=k_{p} t^{n}
$$

$Q$ in the model equation represents the amount of the active compound released as function of time $t$ ( $\mathrm{min})$, $k_{p}$ is a rate constant, and $n$ is the diffusional exponent, indicative of the encapsulated compound release mechanism.

In this mathematical study, the Korsmeyer-Peppas model was used with the aim to determine the release mechanism of the encapsulated compound between three expected mechanisms, Fickian release (diffusion-controlled release), non-Fickian release (anomalous transport), and case II transport (relaxation-controlled release) [20, 21].

The Korsmeyer-Peppas model was particularly established in order to study the release mechanism of substance encapsulated on a polymeric matrix [20], which is the case in this study. The use of the Korsmeyer-Peppas model requires the determination of exponent $n$; accordingly, it is suggested to use only the first $60 \%$ of the substance release data. The $n$ value determined generally used to distinguish between the three mechanisms issued previously, in which Fickian release is verified at the $n$ value less than 0.43 , while the nonFickian release mechanism is established where the $n$ value is between 0.43 and 0.85 , although the $n$ value higher than 0.85 indicates the case II transport release [20-22]. Using OriginPro 2018 software, the calculation of the squared correlation coefficient $\left(R^{2}\right)$ was used to confirm the accuracy of those models.

\section{Results and Discussion}

3.1. Bioactive Substances Characteristics. The calculated ground state geometries of gallic acid and crocin molecules are illustrated in Figure 2. The crocin molecule has a branched and flexible structure due to the conjugated system elongated along the entire polyene chain, whereas the molecule of gallic acid has a well-defined structure.

The obtained physical-chemical characteristics of gallic and crocin bioactive molecules (Table 1) show that crocin is freely soluble in water, while the solubility of gallic acid is limited $(11.9 \mathrm{~g} / \mathrm{l})$, and the polarizability of crocin molecule is higher than of the molecule of gallic acid. In addition, the crocin molecule has a higher topological polar surface area and molecular volume compared with the gallic acid molecule, which allows us to discuss the porosity of our delivery system.

\subsection{Bioactive Substance Content Measurement and Loading} Efficiency. The obtained encapsulation efficiency (EE) and loading capacity (LC) for all elaborated formulations are presented in Table 2. Considering the applied encapsulation method, the used polymer, the low molecular weight, gallic acid solubility in water, and therefore the high solubility of crocin in water, the LC and EE values were satisfying. Moreover, and as shown within the release study, a crucial amount of the encapsulated bioactive substance was released within the primary 20 minutes for gallic acid, and within the 50 minutes for crocin, which suggests that in the preparing process, bioactive substance could release into the solution and, consequently, may limit the loading efficiency. Similar results were reported $[6,8,23,24]$. The results also show that increasing the alginate polymer concentration causes a rise of encapsulation efficiency and a decrease of the loading capacity. This verifies our hypothesis that suggests that the increase of the sodium alginate concentration can certainly lead to introduce a higher level of $\mathrm{Ca}^{2+}$ ions responsible of the cross-linking between the alginate chains and, consequently, forms a dense cohesive structure (heavy microcapsules). The structural properties of the crocin molecule are responsible for the enhancement of EE and LC compared with the encapsulation efficiency and loading capacity of gallic acid (Table 2).

\subsection{Attenuated Total Reflection Fourier Transform Infrared} (ATR-FTIR) Analysis. ATR-FTIR spectra of sodium alginate polymer, gallic acid, blank calcium alginate microcapsules, calcium alginate microcapsules loaded gallic acid (Alg-3\%), calcium alginate microcapsules loaded gallic acid (Alg-4\%), calcium alginate microcapsules loaded gallic acid (Alg-5\%), and calcium alginate microcapsules loaded gallic acid (Alg6\%) are presented in Figure 3.

The spectrum of gallic acid shows two bands at $3268 \mathrm{~cm}^{-1}$ and $3491 \mathrm{~cm}^{-1}$ attributed to the $\mathrm{O}-\mathrm{H}$ function and two other bands situated at $1605 \mathrm{~cm}^{-1}$ and $1307 \mathrm{~cm}^{-1}$ characterize the $\mathrm{C}=\mathrm{O}$ group. Furthermore, the peak at $1021.12 \mathrm{~cm}^{-1}$ with relatively high intensity was assigned to the benzene ring vibration (Figure 3(a)) $[6,25]$. In the case of sodium alginate polymer, the spectrum reveals the presence 


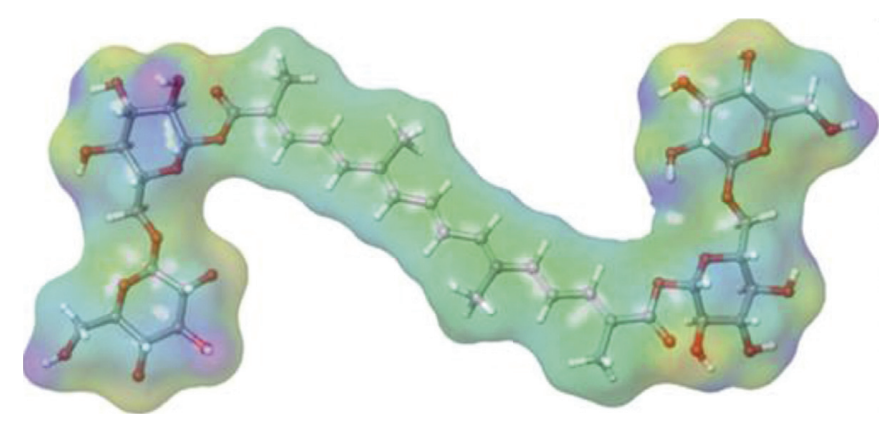

(a)

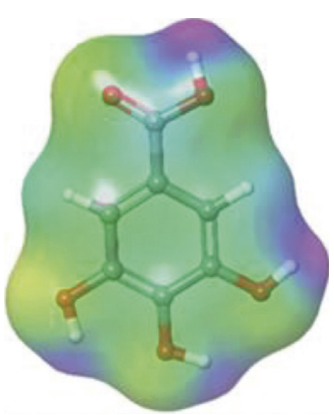

(b)

Figure 2: The optimized geometries of bioactive substances (a) crocin and (b) gallic acid.

TABLE 1: Physical-chemical property of bioactive substances.

\begin{tabular}{|c|c|c|c|c|c|c|}
\hline \multicolumn{2}{|c|}{$\begin{array}{c}\text { Bioactive substances } \\
\text { characteristics }\end{array}$} & $\begin{array}{l}\text { Solubility in water } \\
(\mathrm{mg} / \mathrm{mL}) \text { at } 25^{\circ} \mathrm{C}\end{array}$ & Polarizability $\left(\AA^{3}\right)$ & $\begin{array}{l}\text { Molecular weight } \\
(\mathrm{g} / \mathrm{mol})\end{array}$ & $\begin{array}{c}\text { Topological polar surface } \\
\text { area }\left(\AA^{2}\right)\end{array}$ & $\begin{array}{c}\text { Molecular } \\
\text { volume }\left(\AA^{3}\right)\end{array}$ \\
\hline Phenolic substance & Gallic acid & 11.9 & 12.040 & 170.12 & 98 & 527.659 \\
\hline Flavonoid substance & Crocin & Freely soluble & 84.2 & 977 & 391 & 2794.926 \\
\hline
\end{tabular}

TABLE 2: Encapsulation efficiency and loading capacity of calcium alginate microcapsules.

\begin{tabular}{|c|c|c|c|c|}
\hline \multirow{2}{*}{ Alginate weight (w/v) (\%) } & \multicolumn{2}{|r|}{ Gallic acid } & \multicolumn{2}{|r|}{ Crocin } \\
\hline & $\mathrm{EE}(\%)^{\mathrm{a}}$ & LC (g BS/100 g capsules) ${ }^{\mathrm{a}}$ & $\mathrm{EE}(\%)^{\mathrm{a}}$ & LC (g BS/100 g capsules) ${ }^{\mathrm{a}}$ \\
\hline 3 & 41 & 3.76 & 64.11 & 13.98 \\
\hline 4 & 41.40 & 3.52 & 64.34 & 11.01 \\
\hline 5 & 42.92 & 3.22 & 65.10 & 10.09 \\
\hline 6 & 43.10 & 2.81 & 66.30 & 8.93 \\
\hline
\end{tabular}

${ }^{a}$ All the data are presented as the mean of three replications.

of a broad vibrational band located at $3268 \mathrm{~cm}^{-1}$, which is attributed to the stretching of the $\mathrm{O}-\mathrm{H}$ function. The symmetrical and asymmetrical stretching vibrations of $\mathrm{COO}^{-}$groups were observed at $1593.88 \mathrm{~cm}^{-1}$ and $1405.85 \mathrm{~cm}^{-1}$, respectively (Figure $3(\mathrm{~b})$ ) $[6,26-29]$.

On the other hand, the spectrum of the blank calcium alginate microcapsules shows a broad peak at $3342 \mathrm{~cm}^{-1}$ assigned to $\mathrm{H}_{2} \mathrm{O}$ molecules. Additionally, for the two peaks of $\mathrm{COO}^{-}$groups, a remarked shift toward higher wavenumber was observed $\left(1598 \mathrm{~cm}^{-1}\right.$ and $1435 \mathrm{~cm}^{-1}$ for the symmetrical and asymmetrical vibrations, respectively), suggesting that $\mathrm{Ca}^{2+}$ crosslinked the $\mathrm{COO}^{-}$groups of the alginate (Figure $3(\mathrm{c})$ ).

In the case of calcium alginate microcapsules loaded with gallic acid (Figures $3(\mathrm{~d})-3(\mathrm{~g})$ ), the spectrum indicates a shift of all characteristic peaks to higher wavenumbers without observing any new peaks in comparison with the spectrum of blank calcium alginate microcapsules, thus indicating the incorporation with a possible interaction of gallic acid with the microcapsules as reported in numerous previous studies $[6,8,30]$. In addition, as stated in several reports, gallic acid tends to autoxidize, when electrospinning zein fibers loaded with gallic acid, to dimer or oligomer structure $[8,31,32]$. By using a similar method, our examination illustrates the significant stability of gallic acid in the obtained calcium alginate microcapsules.

Figure 4 represents the ATR-FTIR spectra of crocin, sodium alginate polymer, blank calcium alginate microcapsules, calcium alginate microcapsules loaded crocin (Alg$3 \%$ ), calcium alginate microcapsules loaded crocin (Alg-4\%), calcium alginate microcapsules loaded crocin (Alg-5\%), and calcium alginate microcapsules loaded crocin (Alg-6\%).

The ATR-FTIR spectrum of crocin shows a centered peak at $3301 \mathrm{~cm}^{-1}$ corresponding to hydrogen bonds of the $\mathrm{O}-\mathrm{H}$ of alcoholic groups. Moreover, the band located at $1694 \mathrm{~cm}^{-1}$ represents the $\mathrm{C}=\mathrm{O}$ stretching vibration, and the intense vibrational band at $1014 \mathrm{~cm}^{-1}$ is attributed to the C-O stretching vibration (Figure 4(a)) $[23,33]$.

After elaboration of calcium alginate microcapsules loaded with crocin, it is observed that the characteristic bands were shifted to higher wavenumber (Figures 4(d)4(g)) compared to the spectrum of blank calcium alginate microcapsules (Figure 4(c)) without observing any new peaks, which indicates the stability of microcapsule caused by the incorporation with a possible interaction between crocin and calcium alginate microcapsules [23]. 


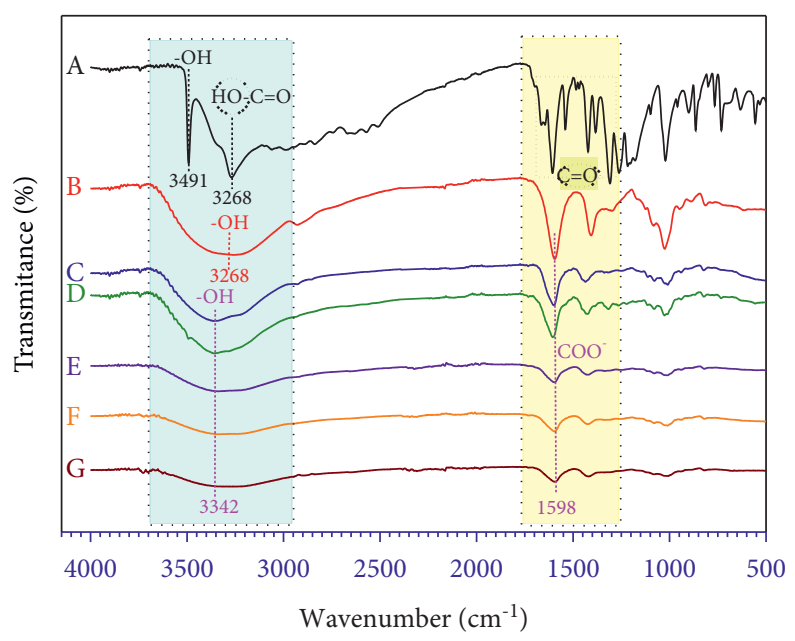

Figure 3: ATR-FTIR spectra of (a) gallic acid, (b) sodium alginate polymer, (c) blank calcium alginate microcapsules, (d) calcium alginate microcapsules loaded gallic (Alg-3\%), (e) calcium alginate microcapsules loaded gallic (Alg-4\%), (f) calcium alginate microcapsules loaded gallic (Alg-5\%), and (g) calcium alginate microcapsules loaded gallic (Alg-6\%).

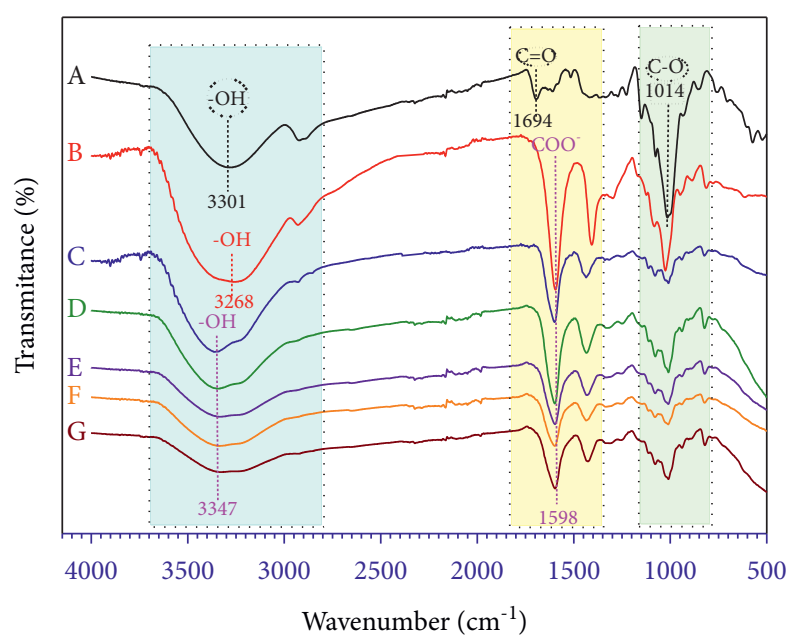

FIGURE 4: ATR-FTIR spectra of (a) crocin, (b) sodium alginate polymer, (c) blank calcium alginate microcapsules, (d) calcium alginate microcapsules loaded crocin (Alg-3\%), (e) calcium alginate microcapsules loaded crocin (Alg-4\%), (f) calcium alginate microcapsules loaded crocin (Alg-5\%), and (g) calcium alginate microcapsules loaded crocin (Alg-6\%).

3.4. XRD Analysis. The diffractograms in Figure 5 show the XRD patterns of sodium alginate powder (Na-Alg), calcium alginate microcapsules (Alg-3\%, w/v), and calcium alginate microcapsules (Alg-5\%, w/v). XRD analysis is performed to study the purity, phase formation, and structural properties of the elaborated formulations. As shown in Figure 5, the XRD spectrum of the sodium alginate $(\mathrm{Na}-\mathrm{Alg})$ indicates an amorphous polymeric structure with a low crystallinity observed by broads peaks at $2 \theta=13.8^{\circ}$ and $21.9^{\circ}$. Also, the obtained microcapsules for different alginate polymer concentrations are amorphous in nature when compared to the sodium alginate pattern. These results are supported by previous studies, which mentioned that the calcium ion crosslinked to the polymeric chains reduces the crystallinity of the elaborated material [34-37].
3.5. Morphological Characterization. Scanning electron microscopy (SEM) analysis was used to investigate the external morphology of the elaborated calcium alginate microcapsules. The collected micrographs presented in Figure 6 show that the blank calcium alginate microcapsules (Alg-3\%, w/v) (Figure 6(a)) appeared with a spherical, smooth, and homogenized surface due to the higher density of crosslinked polymer matrices at the surface and inside of the microcapsules. The microparticles are regularly sized around $941.8 \mu \mathrm{m}$.

The calcium alginate microparticles loaded with gallic acid (Alg-3\%, w/v) (Figure 6(b)) appeared spherical with a wrinkled surface and uniformly sized microcapsules along with a regular surface texture. In high magnification, a regular alignment of "lines" appears on the surface 


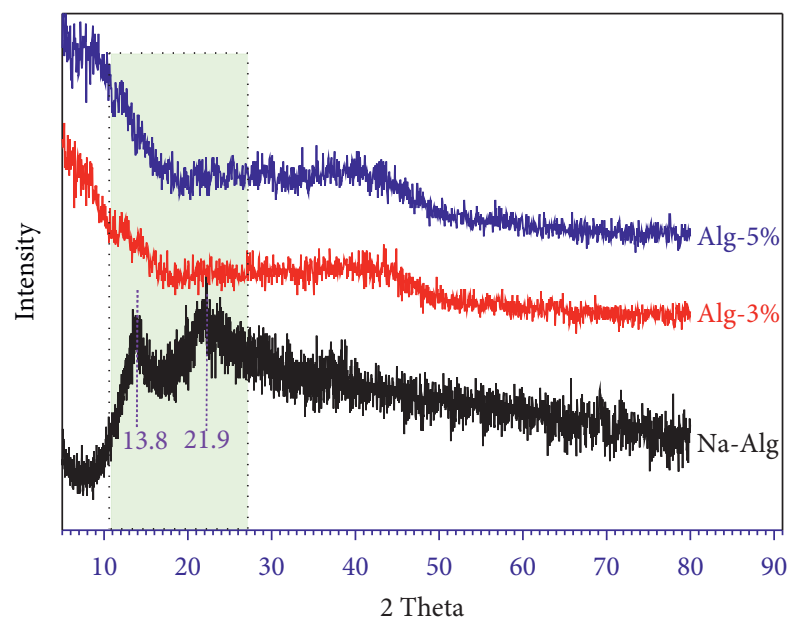

FIGURE 5: XRD patterns of sodium alginate (Na-Alg) and calcium alginate microcapsules loaded gallic acid: Alg-3\% and Alg-5\%.
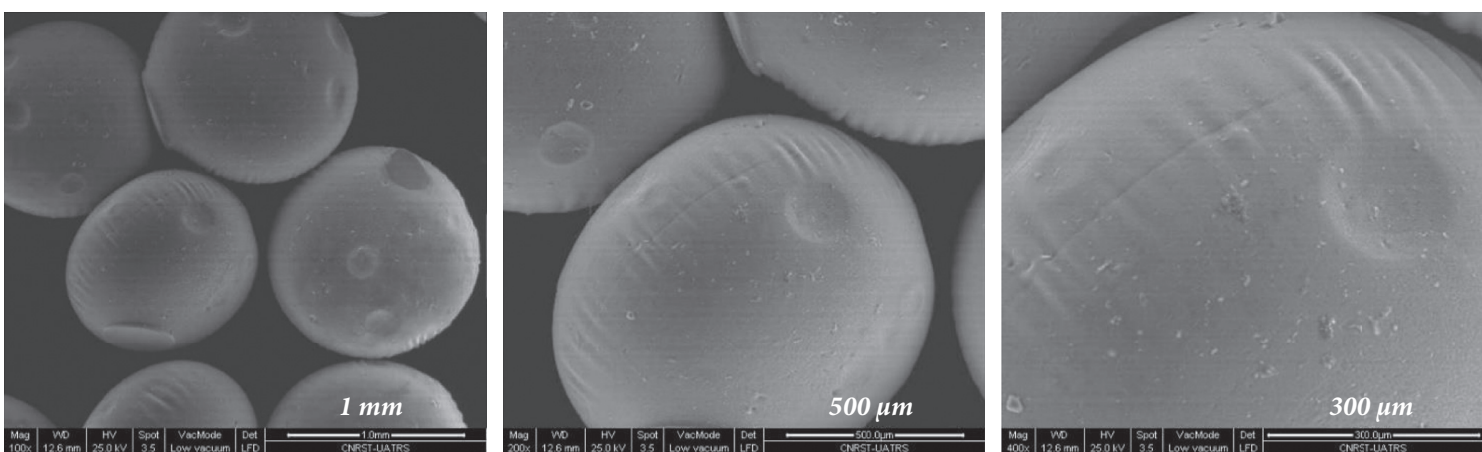

(a)
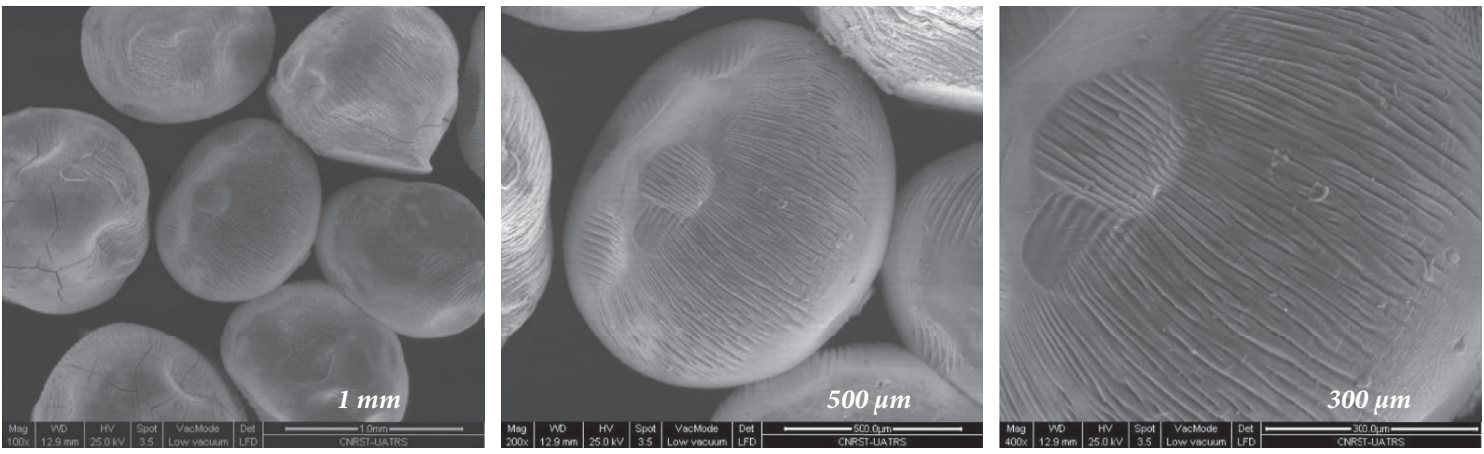

(b)
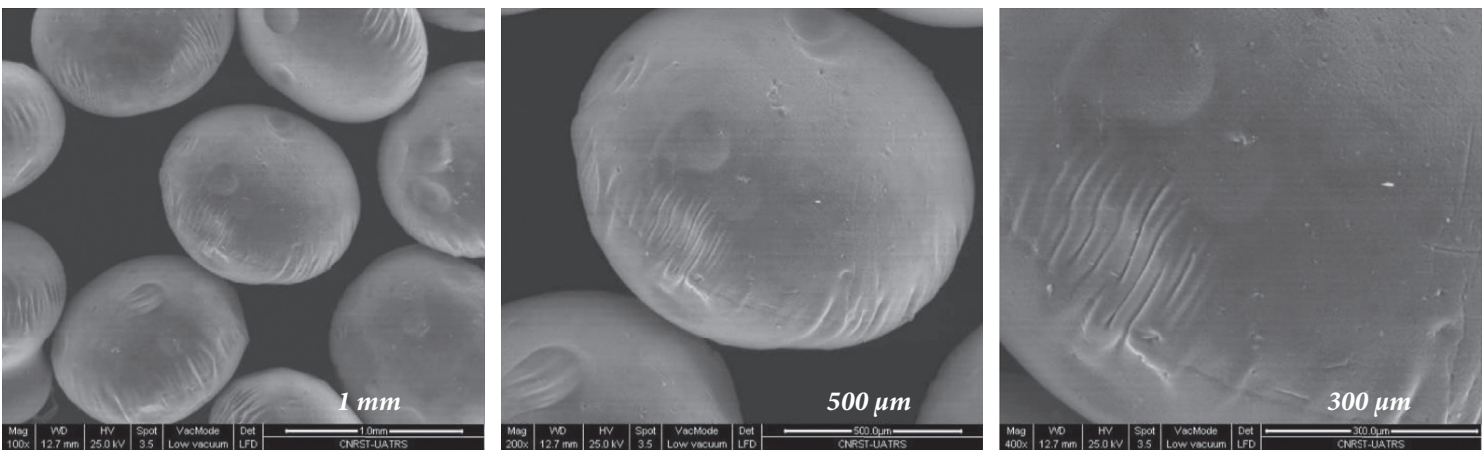

(c)

FIGURE 6: SEM photographs of blank calcium alginate microcapsules Alg-3\% (a) and calcium alginate microcapsules loaded gallic acid (b) Alg-3\% and (c) Alg-5\%. 


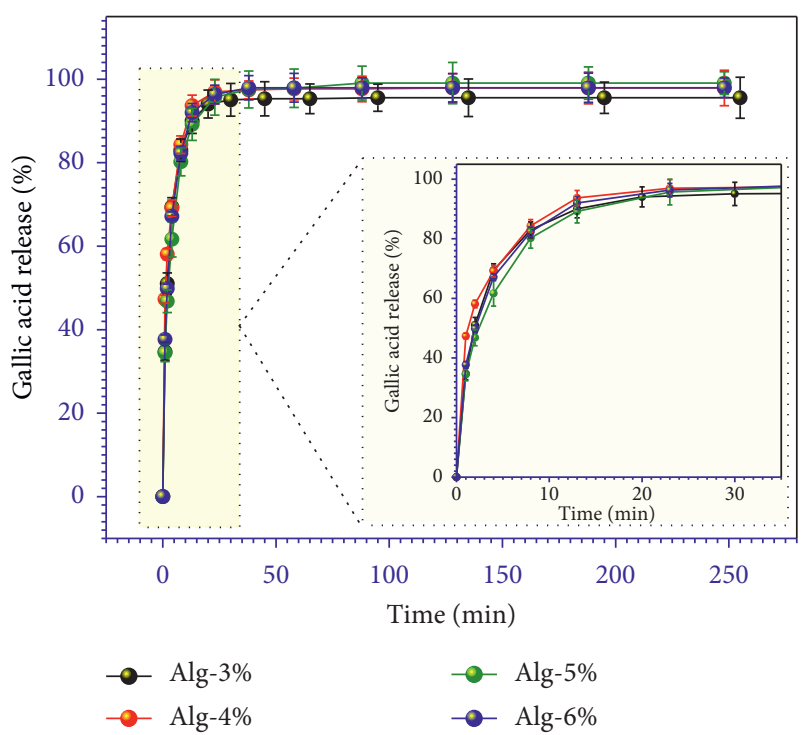

(a)

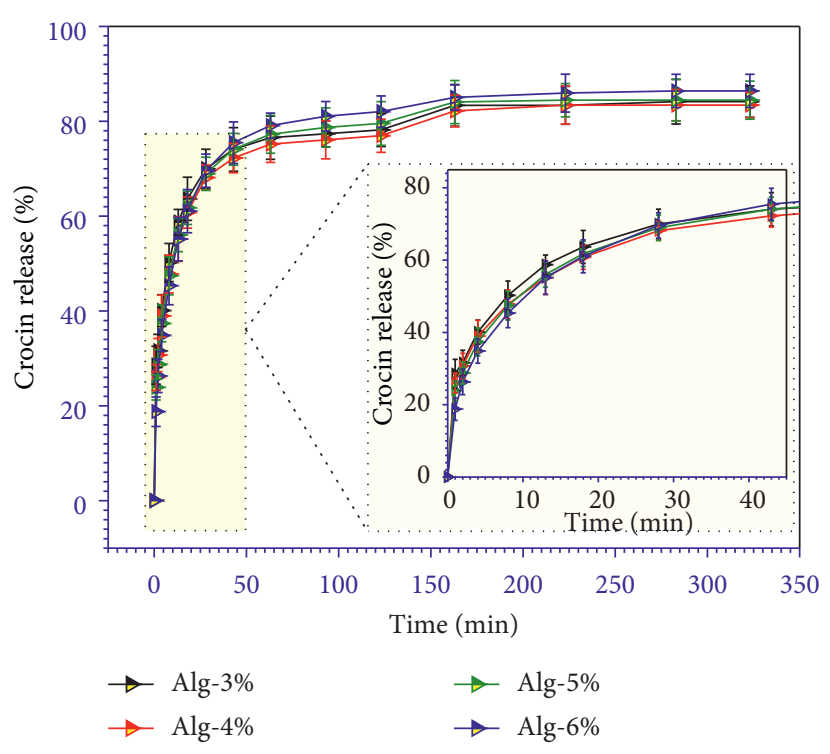

(b)

FIgURE 7: Effect of sodium alginate concentration on the kinetics release of the gallic acid (a) and crocin (b) from calcium alginate microcapsules in distilled water at $\mathrm{pH}=6.8$.

corresponding to the polymer chains; this is due to the low density of crosslinked polymer matrices in $3 \%(\mathrm{w} / \mathrm{v}) \mathrm{mi}-$ crocapsules, resulting from the incorporation of gallic acid molecules and, consequently, a decrease on calcium ions inside the capsules. On the other hand, the microcapsules loaded with gallic acid obtained from $5 \%(\mathrm{w} / \mathrm{v})$ alginate solution have a smoother surface compared to the $3 \%(\mathrm{w} / \mathrm{v})$ microcapsules (Figure 6(c)) due to the higher density of crosslinked polymer matrices at the surface and inside of the capsules.

3.6. Release Study of Bioactive Substances. Figure 7 represents the kinetics release of the bioactive substances from calcium alginate microcapsules elaborated for various concentrations of sodium alginate in distilled water at $25^{\circ} \mathrm{C}$. In the release profile graphs, two stages can be distinguished for all studied systems.

The first stage showed that an initial fast BS release reached $85 \%$ of gallic acid during the first 20 minutes (Figure $7(\mathrm{a})$ ) and $75 \%$ during the 50 minutes for crocin (Figure 7(b)), which can be attributed to the appearance of bioactive substance (BS) on the surface of the microcapsules or close to it (superficial BS). Similar results have been reported $[8,38]$. Generally, calcium alginate microparticles encapsulate hydrophilic substance or water extracts usually have a fast release behavior due to their porous structure which allows the release. In this level of release, the microcapsule does not act like a barrier to prevent the encapsulated compound from being released [39, 40]. In the second stage, the release was characterized by a lower and progressive release rate of the encapsulated bioactive substance. This step was attributed to the release of the encapsulated bioactive substance from the core of calcium alginate microcapsules [8]. At this phase of release, the microcapsules have a higher stability (the erosion of the polymer matrix was not observed), which could be explained by the short time of the release study and the environmental conditions of the release medium.

The effect of sodium alginate concentration on the kinetics release of bioactive substances was not observed, which is explained by the gelling property of the polymer [8]. Generally, an increase of sodium alginate concentration leads to introduce higher levels of $\mathrm{Ca}^{2+}$ ions. Hence, the formulation of a dense and cohesive calcium alginate network. As shown in our previous work [8], sodium alginate polymer concentration lower than $3 \%$ has no effect on the porosity of the microcapsules for the molecules with the same size and physicochemical property as gallic acid molecules; the similar results have been obtained in this work for sodium alginate concentrations, which varies between 3 and $6 \%(\mathrm{w} / \mathrm{v})$. This leads us to conclude that the pores volume of calcium alginate matrix is higher than the molecular volume of gallic acid (total release rate reached 99\%); similar results have been reported [8, 41].

As shown in Figures 8(a)-8(d), the structure of bioactive substance showed some effect on the kinetics release for all obtained formulations; this effect was observed during the release of the encapsulated bioactive substance in the core of the capsules, which is explained by the slow diffusion inside the capsule. The total release rate of gallic acid reached $99 \%$ of the encapsulated amount, while for the crocin molecule, the total release rate reached only $80 \%$ of the encapsulated BS amount during 300 minutes for all obtained formulations (Figures $8(\mathrm{a})-8(\mathrm{~d})$ ). As presented in Table 1 , the crocin molecule is more polar than the gallic acid molecule, which explains the prolonged release of the crocin located on the surface of calcium alginate microcapsules or near to the surface (superficial crocin). This difference on the release rate is mostly due to a strong 


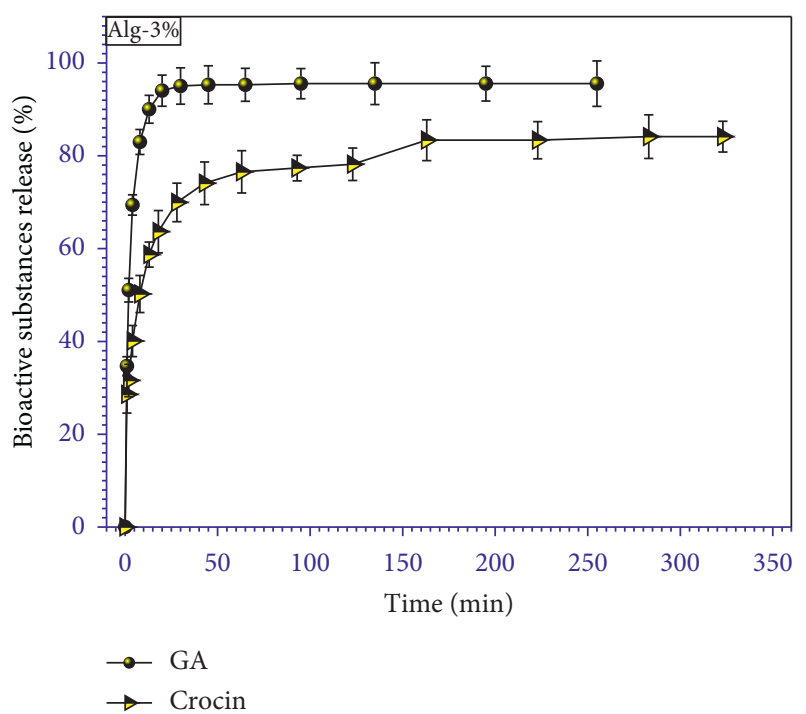

(a)

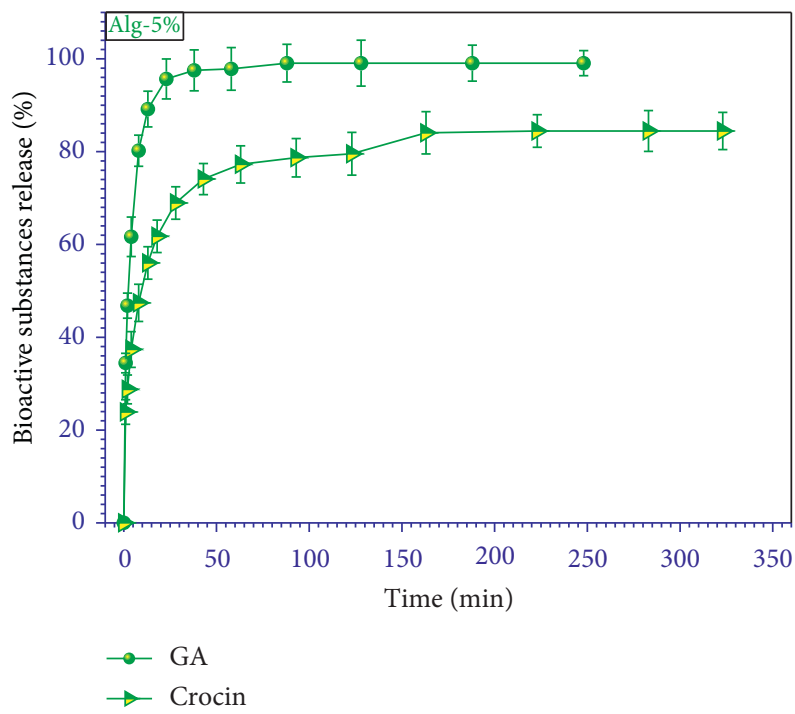

(c)

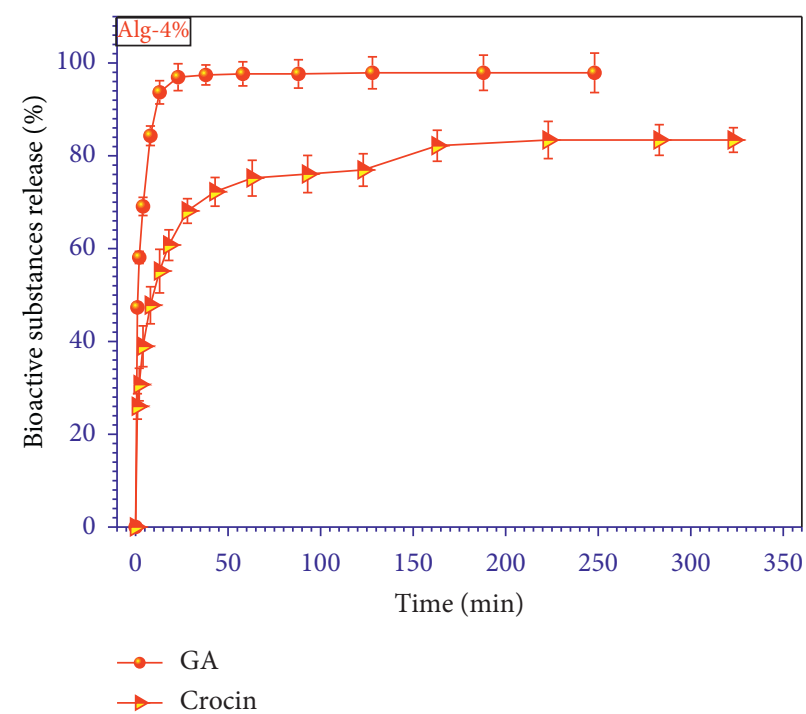

(b)

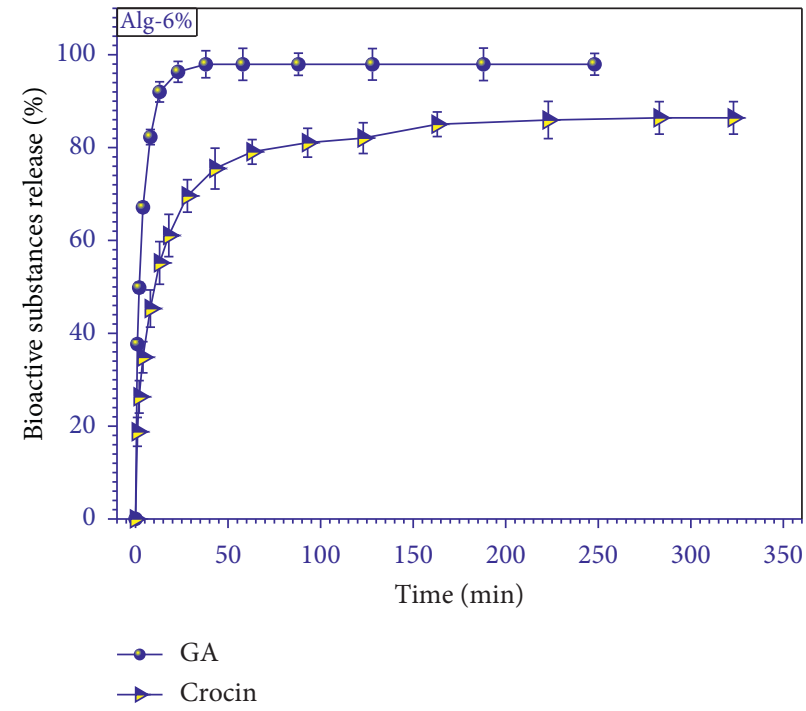

(d)

FIGURE 8: Effect of bioactive substance characteristics on the kinetics release from calcium alginate microcapsules with different sodium alginate concentrations in distilled water at $\mathrm{pH}=6.8$. (a) Alg-3\%; (b) Alg-4\%; (c) Alg-5\%; (d) Alg-6\%.

interaction between crocin molecules and polymeric chains, whereas the gallic acid molecules exhibit a less interaction force with the microcapsule network. Furthermore, the molecular volume of crocin is higher than gallic acid, which clearly explains the limitation of the encapsulated crocin release inside calcium alginate microcapsules. Moreover, we can assume that the pores size of the calcium alginate network is smaller than the volume of crocin molecules. Therefore, the release of the total encapsulated crocin amount needs specific conditions in order to break the calcium alginate network (erosion conditions). Another explanation for the release limitations of crocin is the reticulation between polymeric chains of alginate and crocin molecules due to their ramified structure. The delivery system in this case needs a large period of time to allow the diffusion of crocin molecules through the polymeric network. So, for the same microcapsules system, the release mechanism is affected by the structure and physicochemical properties of the encapsulated molecules; similar results have been reported [42-45].

The kinetics of gallic acid and crocin release were assessed using a variety of mathematical models, including zero-order, first-order, and Korsmeyer-Peppas kinetic models. Using OriginPro 2018 software, the squared correlation coefficient $\left(R^{2}\right)$ values of these models were obtained in order to assess their prediction capacity and accuracy (Figure 9 and Table 3).

Based on the correlation coefficients $\left(R^{2}\right)$, the zero-order and first-order are the worst models. Therefore, the best one was the Korsmeyer-Peppas model, and consequently, the release kinetics of gallic acid and crocin follow the Korsmeyer-Peppas model. 

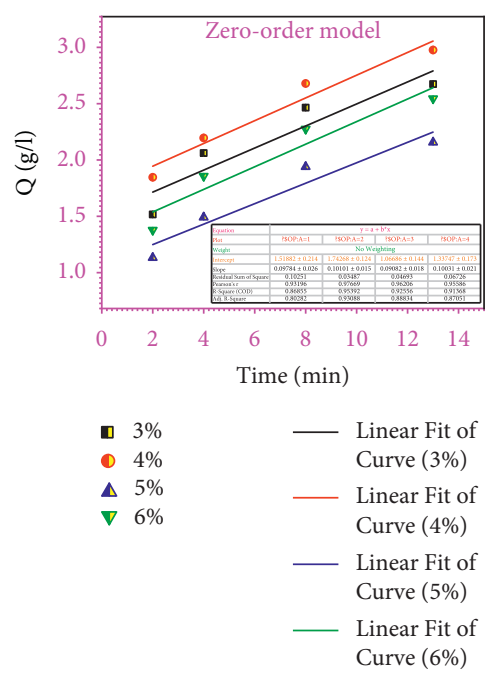

(a)

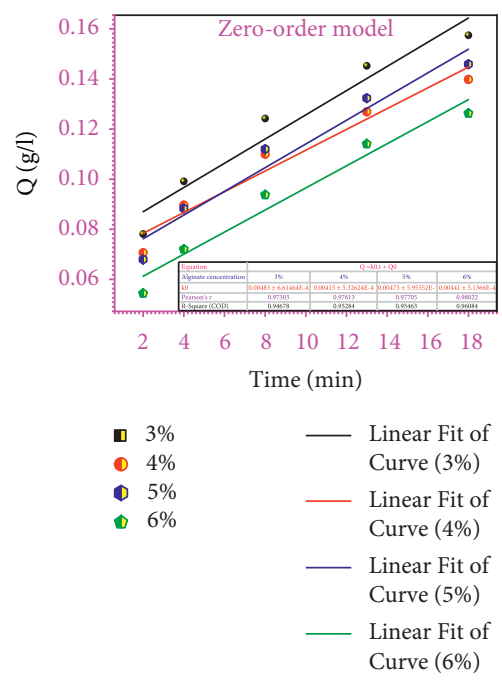

(d)

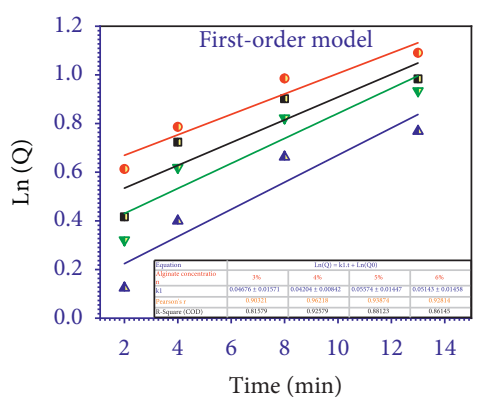

D $3 \%$

- $4 \%$

$\triangle 5 \%$

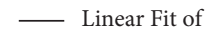
Curve (3\%)

_ Linear Fit of Curve (4\%)

- Linear Fit of Curve (5\%)

Linear Fit of Curve (6\%)

(b)

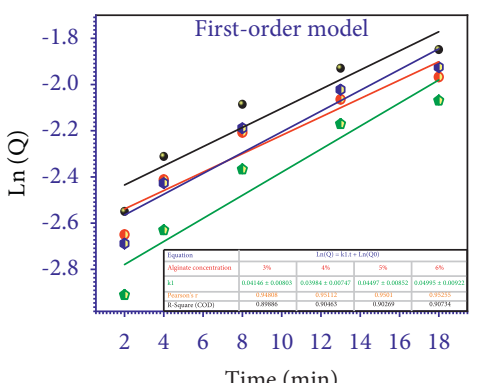

D $3 \% \quad$ Linear Fit of

- $4 \%$

- $5 \%$

- $6 \%$

- Linear Fit of Curve (4\%)

Linear Fit of Curve (5\%)

_ Linear Fit of

Curve (6\%)

(e)

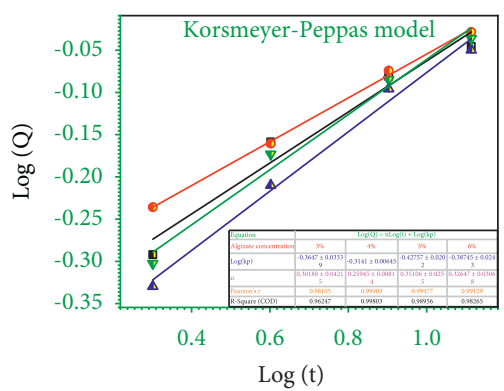

D $3 \%$

- $4 \%$

$\triangle 5 \%$

_ Linear Fit of Curve (3\%)

_ Linear Fit of Curve (4\%)

_ Linear Fit of Curve (5\%)

_ Linear Fit of Curve (6\%)

(c)

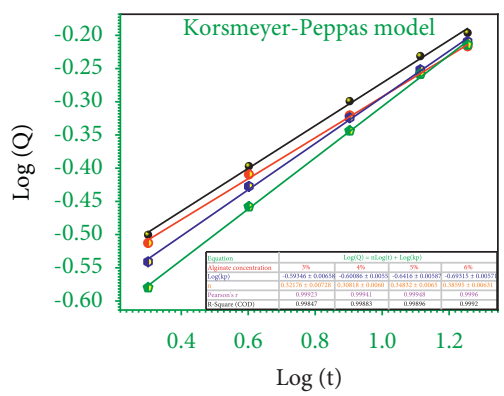

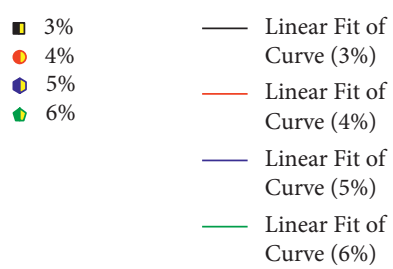

(f)

Figure 9: The fitting of active substance release data to zero-order (a), first-order (b), and Korsmeyer-Peppas (c) kinetic models of the selected sodium alginate concentrations.

TABLE 3: Release kinetics parameters in distilled water at $\mathrm{pH} 6.8$.

Mathematical models

Korsmeyer-Peppas

Microcapsules code (\%)

First-order

Zero-order

Gallic acid

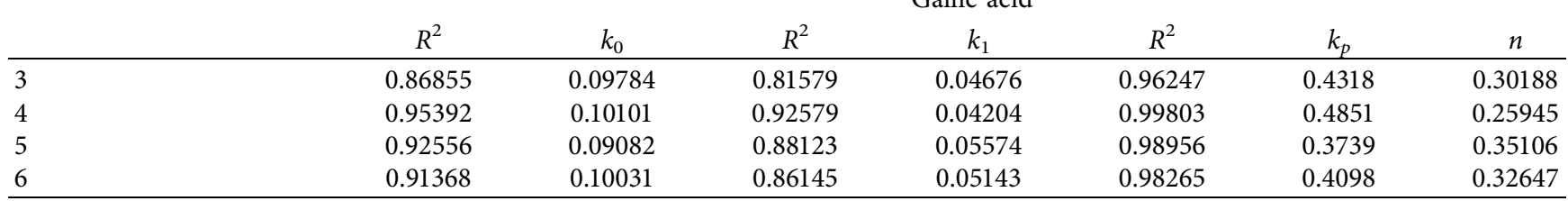

\begin{tabular}{lccccccc}
\hline Microcapsules code (\%) & & & & Crocin & & \\
& $R^{2}$ & $k_{0}$ & $R^{2}$ & $k_{1}$ & $R^{2}$ & $k_{p}$ \\
3 & 0.94678 & 0.00483 & 0.89886 & 0.04146 & 0.99847 & 0.2550 & 0.32176 \\
4 & 0.95284 & 0.00415 & 0.90463 & 0.03984 & 0.99883 & 0.2507 & 0.30818 \\
5 & 0.95463 & 0.00473 & 0.04497 & 0.04497 & 0.99896 & 0.2282 & 0.34832 \\
6 & 0.96084 & 0.00441 & 0.90734 & 0.04995 & 0.9992 & 0.2027 & 0.38595 \\
\hline
\end{tabular}


For all formulation studies, the obtained $n$ values (Table 3) are around $0.310 \pm 0.033$ for gallic acid and $0.341 \pm 0.029$ for crocin, suggesting that the diffusion process of the bioactive substances from the Ca-alginate microcapsules is a Fickian type. Similar results have been found in several reported works: our study about gallic acid release from calcium alginate microcapsules with different sizes [8], the release of cinnamon essential oil encapsulated in alginate beads by Paris et al. [46], and the kinetic release of Trichoderma viride spores loaded calcium alginate microspheres by Jurić et al. [47]. Our results help out to further understand the release mechanisms of phenolic and flavonoid substances such as gallic acid and crocin from Caalginate microcapsules throughout the storage and their use in functional applications.

\section{Conclusion}

As part of the ongoing work, a bioactive substance delivery system based on the calcium alginate network was successfully prepared by the ionotropic gelation method using different concentrations of alginate polymer followed by their physicochemical characterization. Increasing the alginate polymer concentration increases the efficiency of the encapsulation and decreases the carrying capacity. The structure and physicochemical characteristics of the bioactive substance showed a significant effect on the improvement of EE and LC. The ATR-FTIR result confirmed the incorporation of bioactive substances such as gallic acid and crocin in the microcapsules, with a possibility of interaction with the calcium alginate matrix. XRD analysis showed that the low crystallinity observed at sodium alginate polymer disappears when the calcium alginate microcapsule was elaborated. The SEM result showed that the obtained microparticles appeared spherical with a wrinkled surface and a uniform texture.

The release study of gallic acid and crocin as bioactive substances in distilled water (as a hydrophilic system) at $\mathrm{pH}$ value 6.8 showed that the bioactive substances released from microcapsules have fast kinetics release $(85 \%$ within the first 20 minutes for gallic acid and 75\% within the 50 minutes for crocin) with no observed effect of alginate polymer concentration on the release kinetics. Whereas the structure and the physical-chemical properties of the bioactive substance showed a clear effect on the kinetics release for all obtained formulations, this effect could be observed during the release of the encapsulated compounds located in the core of the Caalginate microcapsules. The fitting of the bioactive substances release data to zero-order and first-order, and Korsmeyer-Peppas mathematical models indicates that the diffusion process of the bioactive substances from calcium alginate microcapsules is a Fickian type.

The obtained results suggest that alginate microparticles elaborated by the ionotropic gelling method are a promising system to encapsulate and control the release of watersoluble phenolic and flavonoid compounds such as gallic acid and crocin in order to further increase their applications in functional formulations.
In our future studies, there is a vision to study the effect of polymer structure, polymer-drug ratio, and other parameters on the encapsulation efficiency, loading capacity, release profile, and mechanical property for the simple and hybrid systems (polymer-clay).

\section{Data Availability}

The data used to support the findings of this study are available from the corresponding author upon request.

\section{Conflicts of Interest}

The authors declare that they have no conflicts of interest.

\section{Acknowledgments}

The authors are sincerely thankful to Ministry of National Education, Vocational Training, Higher Education and Scientific Research (MESRSFC), National Center for Scientific and Technical Research (CNRST), Morocco, and University Mohamed I (UMP), Morocco, for financial support of projects PPR 15-17 and PARA1-2019. They would also like to express their gratitude to Prof. El Guennouni Noureddine for his efforts in improving the English.

\section{References}

[1] A. Gray, S. Egan, S. Bakalis, and Z. Zhang, "Determination of microcapsule physicochemical, structural, and mechanical properties," Particuology, vol. 24, pp. 32-43, 2016.

[2] B. Andrade, Z. Song, J. Li et al., "New frontiers for encapsulation in the chemical industry," ACS Applied Materials \& Interfaces, vol. 7, no. 12, pp. 6359-6368, 2015.

[3] K. Essifi, A. Ed-Daoui, D. Berraaouan, M. Benelmostafa, M. Dahmani, and A. Tahani, "Determination of the mechanical properties of single calcium alginate microbeads loaded gallic acid," Materials Today: Proceedings, vol. 31, 2020.

[4] F. Lotfipour, S. Mirzaeei, and M. Maghsoodi, "Preparation and characterization of alginate and psyllium beads containing Lactobacillus acidophilus," Science World Journal, vol. 2012, Article ID 680108, 8 pages, 2012.

[5] H. F. Sakhanokho, C. T. Pounders, and E. K. Blythe, "Alginate encapsulation of begonia microshoots for short-term storage and distribution," Science World Journal, vol. 2013, Article ID 341568, 7 pages, 2013.

[6] J. Li, S. Y. Kim, X. Chen, and H. J. Park, "Calcium-alginate beads loaded with gallic acid: preparation and characterization," LWT-Food Science and Technology, vol. 68, pp. 667-673, 2016.

[7] O. Aarstad, B. L. Strand, L. M. Klepp-Andersen, and G. SkjåkBræk, "Analysis of G-block distributions and their impact on gel properties of in vitro epimerized mannuronan," Biomacromolecules, vol. 14, no. 10, pp. 3409-3416, 2013.

[8] K. Essifi, M. Lakrat, D. Berraaouan, M. Fauconnier, A. El Bachiri, and A. Tahani, "Optimization of gallic acid encapsulation in calcium alginate microbeads using box-behnken experimental," Polymer Bulletin, 2020.

[9] D. Berraaouan, M. Elmiz, S. Salhi, and A. Tahani, "Effect of calcium chloride on rheological behavior of sodium alginate," 
Advanced Materials Proceedings, vol. 2, no. 10, pp. 629-633, 2017.

[10] M. H. L. Ribeiro, C. Afonso, H. J. Vila-Real, A. J. Alfaia, and L. Ferreira, "Contribution of response surface methodology to the modeling of naringin hydrolysis by naringinase Ca-alginate beads under high pressure," LWT-Food Science and Technology, vol. 43, no. 3, pp. 482-487, 2010.

[11] A. Shilpa, S. S. Agrawal, and A. R. Ray, "Controlled delivery of drugs from alginate matrix," Journal of Macromolecular Science: Part C: Polymer Reviews, vol. 43, no. 2, pp. 187-221, 2003.

[12] B. Zeeb, A. H. Saberi, J. Weiss, and D. J. McClements, "Formation and characterization of filled hydrogel beads based on calcium alginate: factors influencing nanoemulsion retention and release," Food Hydrocolloids, vol. 50, pp. 27-36, 2015.

[13] S. Ko and S. Gunasekaran, "Controlled release of food ingredients," in Nano-and Microencapsulation for Foods, pp. 325-343, John Wiley and Sons, Ltd., Hoboken, NJ, USA, 2014.

[14] D. J. McClements, "Encapsulation, protection, and delivery of bioactive proteins and peptides using nanoparticle and microparticle systems: a review," Advances in Colloid and Interface Science, vol. 253, pp. 1-22, 2018.

[15] D. J. McClements, "Encapsulation, protection, and release of hydrophilic active components: potential and limitations of colloidal delivery systems," Advances in Colloid and Interface Science, vol. 219, pp. 27-53, 2015.

[16] A. Matalanis, O. Grif, and D. J. Mcclements, "Structured biopolymer-based delivery systems for encapsulation, protection, and release of lipophilic compounds," Food Hydrocolloids, vol. 25, 2011.

[17] D. McClements, "Particle characteristics and their impact on physicochemical properties of delivery systems," in Nanoparticle-and Microparticle-Based Delivery Systems, pp. 79-122, CRC Press, Boca Raton, FL, USA, 2014.

[18] M. Andersson Trojer, L. Nordstierna, M. Nordin, M. Nydén, and K. Holmberg, "Encapsulation of actives for sustained release," Physical Chemistry Chemical Physics, vol. 15, no. 41, pp. 17727-17741, 2013.

[19] D. Samaha, R. Shehayeb, and S. Kyriacos, "Modeling and comparison of dissolution profiles of diltiazem modifiedrelease formulations," Dissolution Technologies, vol. 16, no. 2, pp. 41-46, 2009.

[20] A. Lamprecht, H. Yamamoto, H. Takeuchi, and Y. Kawashima, "Microsphere design for the colonic delivery of 5-fluorouracil," Journal of Controlled Release, vol. 90, no. 3, pp. 313-322, 2003.

[21] J. d. L. Souza, C. G. Chiaregato, and R. Faez, "Green composite based on PHB and montmorillonite for KNO3 and NPK delivery system," Journal of Polymers and the Environment, vol. 26, no. 2, pp. 670-679, 2018.

[22] S. Dash, P. N. Murthy, L. Nath, and P. Chowdhury, "Kinetic modeling on drug release from controlled drug delivery systems," Acta Poloniae Pharmaceutica-Drug Research, vol. 67, pp. 217-223, 2010.

[23] S. Rahaiee, S. A. Shojaosadati, M. Hashemi, S. Moini, and S. H. Razavi, "Improvement of crocin stability by biodegradeble nanoparticles of chitosan-alginate," International Journal of Biological Macromolecules, vol. 79, pp. 423-432, 2015.

[24] J. Li, H. Wu, K. Jiang, Y. Liu, L. Yang, and H. J. Park, “Alginate calcium microbeads containing chitosan nanoparticles for controlled insulin release," Applied Biochemistry and Biotechnology, vol. 193, no. 2, pp. 463-478, 2020.

[25] H. Hu, L. Nie, S. Feng, and J. Suo, "Preparation, characterization and in vitro release study of gallic acid loaded silica nanoparticles for controlled release," Die Pharmazie, vol. 68, pp. 401-405, 2013.

[26] J. Zhao, S. Li, Y. Zhao, and Z. Peng, "Effects of cellulose nanocrystal polymorphs and initial state of hydrogels on swelling and drug release behavior of alginate-based hydrogels," Polymer Bulletin, vol. 77, no. 8, 2019.

[27] A. Salisu, M. M. Sanagi, A. Abu Naim, K. J. Abd Karim, W. A. Wan Ibrahim, and U. Abdulganiyu, "Alginate graft polyacrylonitrile beads for the removal of lead from aqueous solutions," Polymer Bulletin, vol. 73, no. 2, pp. 519-537, 2016.

[28] S. Peretz, D. F. Anghel, E. Vasilescu, M. Florea-Spiroiu, C. Stoian, and G. Zgherea, "Synthesis, characterization and adsorption properties of alginate porous beads," Polymer Bulletin, vol. 72, no. 12, pp. 3169-3182, 2015.

[29] J. Li, I. W. Lee, G. H. Shin, X. Chen, and H. J. Park, "Curcumin-eudragit ${ }^{\circledR}$ E PO solid dispersion: a simple and potent method to solve the problems of curcumin," European Journal of Pharmaceutics and Biopharmaceutics, vol. 94, pp. 322-332, 2015.

[30] A. López Córdoba, L. Deladino, and M. Martino, "Effect of starch filler on calcium-alginate hydrogels loaded with yerba mate antioxidants," Carbohydrate Polymers, vol. 95, no. 1, pp. 315-323, 2013.

[31] P. N. Yun, S. Simon, R. Sudip, G. N. Marija, J. Jianyong, and O. P. Conrad, "Evaluation of gallic acid loaded zein submicron electrospun fibre mats as novel active packaging materials," Food Chemistry, vol. 141, pp. 3192-3200, 2013.

[32] P. N. Yun, R. Sudip, J. Jianyong et al., "Encapsulation of food grade antioxidant in natural biopolymer by electrospinning technique: a physicochemical study based on zein-gallic acid system," Food Chemistry, vol. 136, pp. 1013-1021, 2013.

[33] F. Y. Lee, T. T. Htar, and G. A. Akowuah, "ATR-FTIR and spectrometric methods for the assay of crocin in commercial saffron spices (crocus savitus L.)," International Journal of Food Properties, vol. 18, no. 8, pp. 1773-1783, 2015.

[34] E. Kolanthai, P. A. Sindu, D. K. Khajuria et al., "Graphene oxide-a tool for the preparation of chemically crosslinking free alginate-chitosan-collagen scaffolds for bone tissue engineering," ACS Applied Materials \& Interfaces, vol. 10, no. 15, pp. 12441-12452, 2018.

[35] R. Zhang, L. Lei, Q. Song, and X. Li, "Calcium ion crosslinking alginate/dexamethasone sodium phosphate hybrid hydrogel for extended drug release," Colloids and Surfaces B: Biointerfaces, vol. 175, pp. 569-575, 2019.

[36] Y. D. Yashaswini, A. Prabhu, S. Anil, and J. Venkatesan, "Preparation and characterization of dexamethasone loaded sodium alginate-graphene oxide microspheres for bone tissue engineering," Journal of Drug Delivery Science and Technology, vol. 64, Article ID 102624, 2021.

[37] M. Ionita, M. A. Pandele, and H. Iovu, "Sodium alginate/ graphene oxide composite films with enhanced thermal and mechanical properties," Carbohydrate Polymers, vol. 94, no. 1, pp. 339-344, 2013.

[38] R. Paz, G. Paula, N. Reyes, J. Chávez, and J. Santos, “Acetylated starch and inulin as encapsulating agents of gallic acid and their release behaviour in a hydrophilic system," Food Chemistry, vol. 134, pp. 1-8, 2012.

[39] C. Ross, W. Rangika, S. Luz, and A. MaryAnn, "Synbiotic microcapsules that enhance microbial viability during 
nonrefrigerated storage and gastrointestinal transit," Applied and Environmental Microbiology, vol. 72, pp. 2280-2282, 2006.

[40] B. Lupo, A. Maestro, J. M. Gutiérrez, and C. González, "Characterization of alginate beads with encapsulated cocoa extract to prepare functional food: comparison of two gelation mechanisms," Food Hydrocolloids, vol. 49, pp. 25-34, 2015.

[41] M. Hu, G. Zheng, D. Zhao, and W. Yu, "Characterization of the structure and diffusion behavior of calcium alginate gel beads," Journal of Applied Polymer Science, vol. 137, pp. 1-9, 2020.

[42] J. M. C. Puguan, X. Yu, and H. Kim, "Diffusion characteristics of different molecular weight solutes in Ca-alginate gel beads," Colloids and Surfaces A: Physicochemical and Engineering Aspects, vol. 469, pp. 158-165, 2015.

[43] Q. Pan, X. Deng, W. Gao, J. Chang, Y. Pu, and B. He, "Small molecules-PEG amphiphilic conjugates as carriers for drug delivery: 1. the effect of molecular structures on drug encapsulation," Journal of Drug Delivery Science and Technology, vol. 60, Article ID 101997, 2020.

[44] L. Rolland, E. Santanach-Carreras, T. Delmas, J. Bibette, and N. Bremond, "Physicochemical properties of aqueous core hydrogel capsules," Soft Matter, vol. 10, no. 48, pp. 9668-9674, 2014.

[45] G. S. Offeddu, E. Axpe, B. A. C. Harley, and M. L. Oyen, "Relationship between permeability and diffusivity in polyethylene glycol hydrogels," AIP Advances, vol. 8, Article ID 105006, 2018.

[46] M. J. Paris, N. Ramírez-Corona, E. Palou, and A. López-Malo, "Modelling release mechanisms of cinnamon (Cinnamomum zeylanicum) essential oil encapsulated in alginate beads during vapor-phase application," Journal of Food Engineering, vol. 282, 2020.

[47] S. Jurić, E. Đermić, S. Topolovec-Pintarić, M. Bedek, and M. Vinceković, "Physicochemical properties and release characteristics of calcium alginate microspheres loaded with Trichoderma viride spores," Journal of Integrative Agriculture, vol. 18, pp. 2534-2548, 2019. 